\title{
Temporal artery biopsies: a fresh perspective
}

A negative biopsy result from a temporal artery with a clinical diagnosis of giant cell arteritis (GCA) has the potential to prevent unnecessary exposure of an older population group to high doses of steroids. Figure 1 and 2 show the typical histopathological changes that occur in GCA. While imaging modalities ${ }^{1-4}$ such as Doppler ultrasound offer a potential non-invasive means of diagnosing the disease, temporal artery biopsies remain the gold standard for diagnosis of GCA. However, the role of the biopsy result in changing management has remained controversial, and some surgeons are reluctant to biopsy suspect arteries.

The age-adjusted incidence rate of GCA in the UK from 1990 to 2001 was 2.2/10 000 person-years. ${ }^{5}$ Temporal artery biopsies are commonly encountered procedures for many surgeons. While it is a minor procedure often performed under a local anaesthetic, it is not without risks and complications. The complication rate is $0.5 \%$ with serious complications such as facial nerve damage, skin necrosis, infection and stroke arising due to interruption of collaterals. ${ }^{6}$ Therefore, a more valid justification for performing this procedure is necessary.

The studies in the literature that have explored this have conflicting results. For example, Chong and Robertson ${ }^{7}$ conducted a fiveyear retrospective analysis of patients who underwent temporal artery biopsy (TAB) at the Royal Melbourne Hospital between 1999 and 2003. The management of $13(23.6 \%)$ patients was influenced by the biopsy result, where seven patients with negative biopsies were discontinued on steroids, and six patients with active or healed arteritis were continued on steroids. Management of $42(76.4 \%)$ patients was not altered following biopsy results: 11 with negative biopsy continued on steroids, 19 never started because of low clinical suspicion and 12 ceased steroids some time after biopsy as there was no symptomatic improvement. The authors concluded that the procedure was worthwhile as it is simple and altered management in $23 \%$ of the patients, most of whom are elderly.

The study by Lenton et al. ${ }^{8}$ found there was no change in the clinical management of $31 / 44(70.5 \%)$ patients who had a negative biopsy result. Eighteen patients continued on steroids for six months despite a negative biopsy result, and for 13 patients, a decision to stop steroids or an alternative diagnosis was made before the biopsy result was obtained. Therefore, the authors have justly questioned the true value of the biopsy result in clinical management.

Similarly, Moutray et al. ${ }^{9}$ found that a large portion (76\%) of their study population who had a clinical diagnosis of GCA had negative biopsies. However, a positive biopsy played an important role in validating the use of long-term steroids in a cohort of patients who present with a constellation of symptoms and signs that may indicate GCA.
The Vascular Surgical Service at The Canberra Hospital performed 54 temporal artery biopsies from 2003 to 2008 (inclusive): $14(26 \%)$ had positive biopsies and were continued on a high dose of prednisone and $37(92.5 \%)$ were weaned off prednisone following a negative biopsy result. Therefore, management of 51 (94.4\%) of the patients was altered by the biopsy result. Management of three

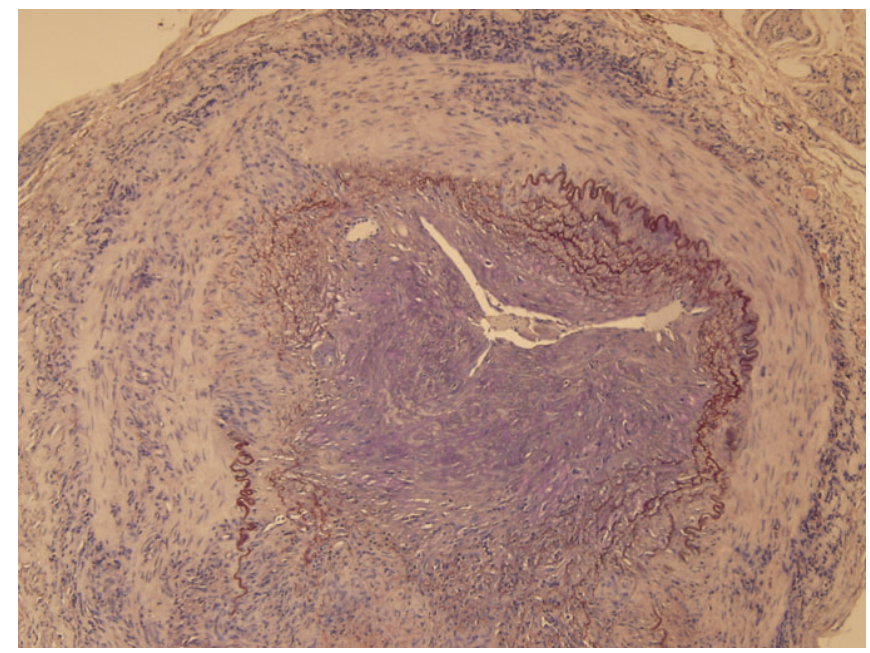

Fig. 1. Cross-section of an artery displaying histopathological changes in all layers of the arterial wall in a patient with GCA. There is mixed infiltrate of neutrophils and lymphocytes in the intima and muscularis layers. There is also fragmentation of the internal elastic lamina.

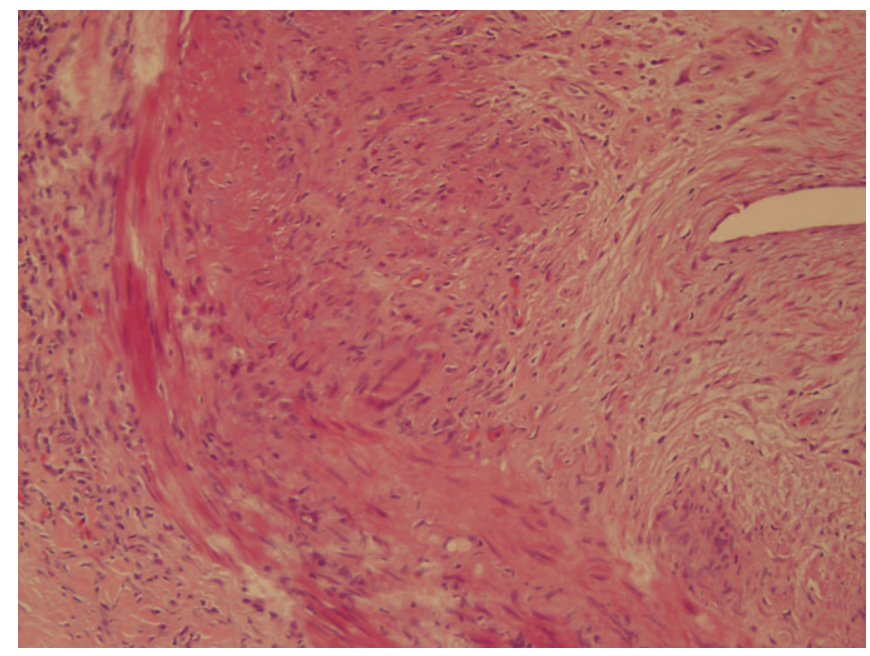

Fig. 2. Higher resolution of the slide shows multinucleate giant cell formation. 
(5.5\%) of the patients was unaltered by the result as they were continued on a high dose of prednisone despite a negative result. These patients were subsequently diagnosed with polymyalgia rheumatica.

The optimum rate of positive biopsies produced in an institution depends on a number of factors: clinical acumen in selecting appropriate patients for a biopsy, patient demographics and availability of services at the institution. The percentage of positive biopsies reported in the literature range from 20 to $82 \% .^{10}$

The American College of Rheumatology (ACR) had developed a five-point scoring system with equal weight for the following criteria: age $>50$ years, erythrocyte sedimentation rate $>50 \mathrm{~mm} / \mathrm{h}$, superficial temporal artery tenderness, temporal headache and positive histology with TAB. ${ }^{11}$ Studies aimed at determining the efficacy of the ACR criteria ${ }^{11,12}$ in directing appropriate selection of patients for a biopsy found that patients who had an ACR score of $\leq 2$ necessitated a biopsy for confirming the diagnosis, whereas for patients who had a score of $\geq 3$, a biopsy added little value. However, majority of the studies in the literature are retrospective in nature and therefore rely on the completeness of medical records. It is often unclear if a standard approach was taken to the clinical diagnosis of GCA and the specific diagnostic criteria that were utilized in selecting a patient for a biopsy.

$\mathrm{TAB}$ is a valuable test that contributes to the management of patients with clinical suspicion of GCA as it delineates between patients who need to remain on high-dose steroids for an extended period of time and those who do not require high-dose steroids, thereby avoiding the adverse side effects. Our study confirms the value of TAB in the management of a difficult-to-identify clinical syndrome, which are patients with GCA.

\section{References}

1. Genereau T, Lortholary O, Guillevin L et al. Temporal gallium uptake is increased in temporal arteritis. Rheumatology 1999; 38: 709-13.
2. Schmidt WA, Gromnica-Ihle E. Incidence of temporal arteritis in patients with polymyalgia rheumatica: a prospective study using colour Doppler ultrasonography of the temporal arteries. Rheumatology 2002; 41: 46-52.

3. Bley TA, Markl M, Schelp M et al. Mural Inflammatory hyperenhancement in MRI of giant cell arteritis resolves under corticosteroid treatment. Rheumatology 2008; 47: 65-7.

4. Fontini B, Karassa MD, Miltiadis I et al. Meta-analysis: test performance of ultrasonography for giant cell arteritis. Ann. of Int. Medicine 2005: 142: 359-69.

5. Smeeth L, Cook C, Hall AJ. Incidence of diagnosed polymyalgia rheumatica and temporal arteritis in the United Kingdom 1990-2001. Ann. Rheum. Dis. 2006; 65: 1093-8.

6. Bhatti MT, Goldstein MH. Facial nerve injury following temporal artery biopsy. Dermatol. Surg. 2001; 27: 15-7.

7. Chong EWT, Robertson AJ. Is temporal artery biopsy a worthwhile procedure? ANZ J. Surg. 2005; 75: 388-91.

8. Lenton J, Donnelly R, Nash JR. Does temporal artery biopsy influence the management of temporal arteritis? QJM 2006; 99: 33-6.

9. Moutray TN, Williams MA, Best JL. Suspected giant cell arteritis: a study of referrals for temporal artery biopsy. Can. J. Ophthalmol. 2008; 43: $445-8$.

10. Davies C, Frost B, Eshan O et al. Temporal artery biopsy ... Who needs one? Postgrad. Med. J. 2006; 82: 476-8.

11. Hunder GG, Bloch DA, Michel BA et al. The American College of Rheumatology 1990 criteria for the classification of giant cell arteritis. Arthritis Rheum. 1990; 33: 1122-8.

12. Varma D, O'Neil D. Quantification of the role of the temporal artery biopsy in diagnosing clinically suspected giant cell arteritis. Eye 2004; 18: $384-8$.

Raj Manjuka, MBBS

Hardman David TA, FRACS

Department of Surgery, Australian National University,

The Canberra Hospital,

Garran, Australian Capital Territory, Australia

doi: 10.1111/j.1445-2197.2010.05361.x

\section{Asymmetric hearing loss in industry}

The guidelines ${ }^{1}$ for evaluation of noise-induced hearing loss (NIHL) suggest that in cases of asymmetry, 'the worse ear be equated to the better ear' for purposes of compensation. This method is prejudicial to the worker (plaintiff). It is my objective in this discussion 'to co-construct the ideas of truth and the ideas of justice in the context of legal proceedings' ${ }^{2}$ in these cases.

The criteria for the diagnosis of occupational NIHL are wellknown. Substantial noise exposure is the sine qua non in the diagnosis. The history and examination by an ear, nose and throat (ENT) expert serves to eliminate other complicating and diagnostic possibilities.

The list of causes of asymmetrical hearing loss is long, and includes cerebello-pontine angle tumours, head injury, viral or vascular deafness, Ménière's disease and perilymphatic fistula. Under these circumstances, the ENT expert is often hesitant to declare that the asymmetry is noise-induced and hence, the guidelines 'statement'.

Although it behoves the ENT doctor to aid the patient in arriving at a diagnosis and symptom alleviation, the ENT expert in a legal situation is not so obligated to the client alone but also to the court. In the latter situation, the ENT has an obligation to assist the court by 'reason of specialized training, study or experience' ${ }^{3}$ and is permitted to offer opinions to the court as to the meaning and implications of other evidence.

The ENT expert must realize that in the legal arena, he or she is governed by the 'preponderance of the evidence' standard - which can be expressed probabilistically as the probability of the facts supporting noise as the causative agent, exceeds 0.5 . This differs 